\title{
Ressecção de tumor ósseo guiado por Gamaprobe e avaliação do quadro álgico pós-operatório*
}

\section{Resection of Bone Tumor Guided by Gamaprobe and Evaluation of Postoperative Pain} \author{
Thércio Murilo Souza Rocha ${ }^{3}$ \\ ${ }^{1}$ Onco-ortopedia, Fundação Hospitalar de Blumenau, Hospital Santo \\ Antônio, Blumenau, SC, Brasil \\ 2 Departamento de Ortopedia e Traumatologia, Hospital Santo \\ Antônio, Blumenau, SC, Brasil \\ ${ }^{3}$ Medicina Nuclear, Clínica Cintilius - Medicina Nuclear, Blumenau, SC, Brasil \\ Rev Bras Ortop 2020;55(1):115-120.
}

Alberto Ramos Gomes ${ }^{1}$ Felipe Younes Quatrin ${ }^{2}$ Nadiane Maciel Becker ${ }^{2}$ Ruan Carlo Zanella2®i)

Endereço para correspondência Ruan Carlo Zanella, Rua Tobias Barreto, 263 - Apartamento 63 - Bairro Vila Nova, Blumenau, SC, 89035-070, Brasil (e-mail: ruan.zanella@gmail.com).

\section{Resumo \\ Palavras-chave \\ - osteoma osteóide/ diagnóstico por imagem \\ - osteoma osteóide/ cirurgia \\ - neoplasias ósseas/ diagnóstico por imagem \\ - neoplasias ósseas/ cirurgia \\ - dor}
Abstract
Keywords
- osteoma, osteoid/ diagnostic imaging
- osteoma, osteoid/ surgery
- bone neoplasms/ diagnostic imaging
- bone neoplasms/ surgery
- pain

O osteoma osteóide é uma neoplasia benigna comum em homens jovens; porém, pode atingir qualquer idade, acomete preferencialmente ossos longos, e é caracterizada por dor contínua e limitante que é aliviada por salicilatos. É uma lesão de tamanho pequeno, composta por tecido osteóide imaturo, hipervascularização central e área esclerótica circundante. O diagnóstico é realizado pela clínica e auxiliado por exames de imagem, como radiografia comum e tomografia computadorizada. À radiografia, apresenta-se como uma lesão radiolucente central e esclerose periférica. A biópsia está indicada somente nos casos de dúvida diagnóstica. O tratamento cirúrgico envolve a ressecção do nicho, proporcionando maior alívio sintomático, assim como as técnicas percutâneas. A técnica de ressecção guiada por radioisótopo tem boa aceitação na comunidade científica por tornar o procedimento cirúrgico mais rápido e dirigido para a lesão, ainda que o padrão ouro de tratamento seja a técnica percutânea de ablação por radiofrequência. É válido ressaltar que, no presente estudo, todos os pacientes submetidos à resecção cirúrgica apresentaram regressão do quadro álgico.

Osteoid osteoma is a benign neoplasm commonly found in young men, but that can be found in every age, which affects mainly long bones, clinically characterized by continuous and limiting pain that is relieved by salicylates. It is a small lesion composed of immature osteoid tissue, central hypervascularization and surrounding sclerotic area. Its diagnosis is performed by the clinic and aided by imaging tests, such as common radiography and computed tomography. Radiography shows a central radiolucent lesion and peripheral sclerosis. The biopsy takes place only in cases of diagnostic doubt. Surgical treatment involves resection of the niche, providing greater symptomatic relief, as well as percutaneous techniques. The technique of radioisotope-guided resection has good acceptance in the scientific community for its fast surgical procedure directed to the lesion; however, the percutaneous technique that stands out is radiofrequency ablation. In the present study, all of the patients submitted to the technique presented total improvement of the pain.

Trabalho feito no Hospital Santo Antonio, Blumenau, SC, Brasil.

recebido

08 de Janeiro de 2018

aceito

30 de Outubro de 2018
DOI https://doi.org/

10.1055/s-0039-1700818. ISSN 0102-3616.
Copyright $(2020$ by Sociedade Brasileira License terms de Ortopedia e Traumatologia. Published by Thieme Revinter Publicações Ltda, Rio de Janeiro, Brazil 


\section{Introdução}

O osteoma osteóide é uma neoplasia benigna, que acomete homens jovens em uma proporção de até 4:1, sendo encontrados, geralmente, na $2^{\underline{a}}$ ou $3^{\text {a }}$ décadas de vida, porém, podem acometer pacientes em qualquer idade. ${ }^{1}$ Trata-se de uma doença autolimitada, com duração média de 3 anos. $^{2}$ Foi descrito originalmente por $\mathrm{Jaffe}^{3}$ em 1935 como um tumor benigno com predileção por ossos longos dos membros inferiores rodeado por um nicho, caracterizado por tecido osteóide imaturo, hipervascularização central, área esclerótica em seu entorno e tamanho pequeno, variando de $1 \mathrm{a} 10 \mathrm{~mm} .{ }^{4,5}$

Apresenta-se clinicamente por dor perene, com maior intensidade noturna, e que por vezes alivia com o uso de salicilatos e anti-inflamatórios não esteroidais (AINEs).

São utilizados como exames complementares para auxílio diagnóstico a radiografia simples (- Figura 1) e a tomografia computadorizada (TC) - padrão ouro (-Figura 2). ${ }^{1,6}$ A cintilografia óssea com fluxo de tecnécio 99 ( - Figura 3) apesenta sensibilidade de $100 \%$ no diagnóstico do osteoma osteoide, sendo o exame que detecta com maior exatidão a localização
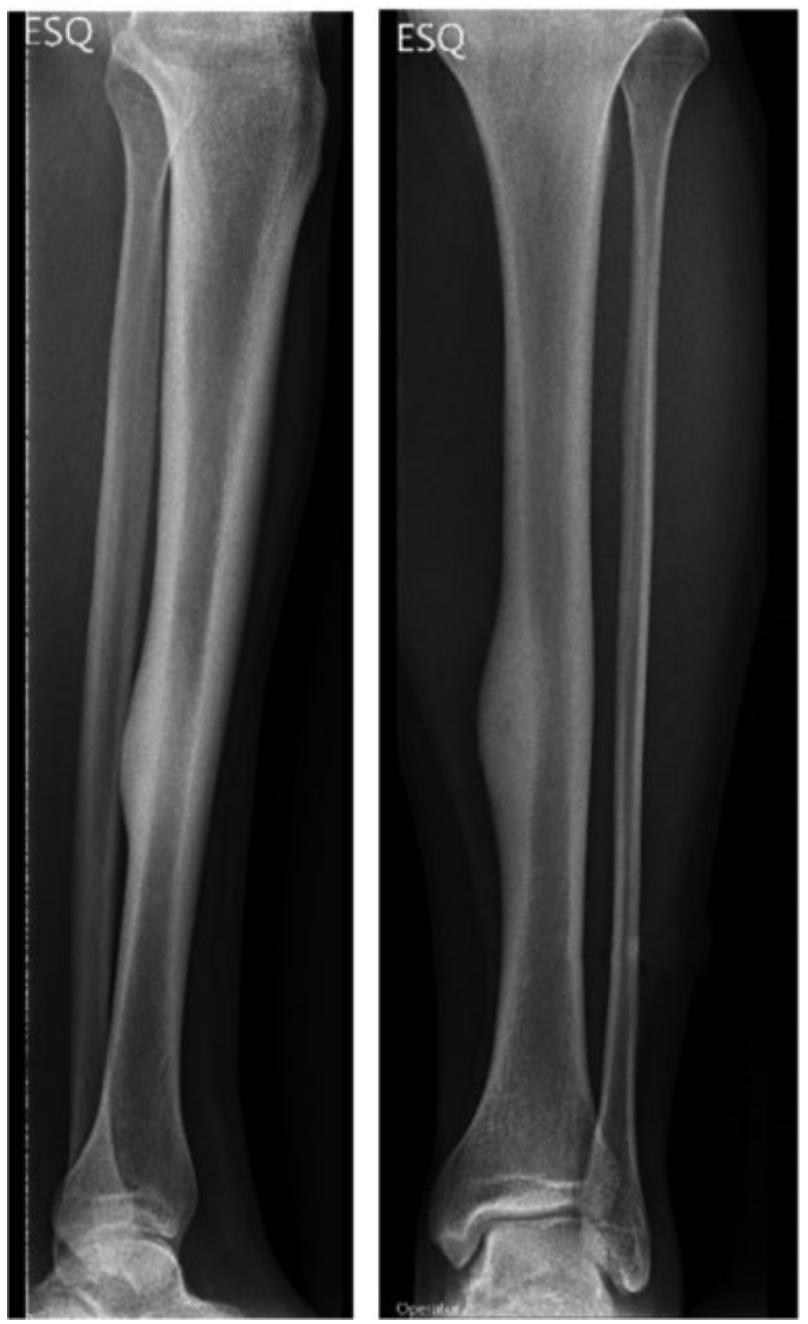

Fig. 1 Radiografia simples anteroposterior e lateral da perna demonstrando área hiperdensa com presença de reação periosteal local, sinais de esclerose local com pequena área hipodensa mais próxima à região da cortical lateral na diáfise da tíbia, podendo corresponder ao nicho. do tumor, devido ao grande aumento da captação do radiofármaco, tornando a biópsia raramente necessária.

Por ser extremamente sensível, a cintilografia confirma o diagnóstico e reduz o atraso na obtenção do mesmo, principalmente nas lesões em estadios precoces, pois as alterações cintigráficas ocorrem antes das radiográficas. Os principais diagnósticos diferenciais para osteoma osteóide são condroblastoma, infarto ósseo e osteomielite crônica.

Macroscopicamente, o nicho pode ser identificado como lesão bem delimitada, de coloração violácea, geralmente circundada por halo esclerótico. Microscopicamente, é constituído por tecido fibrovascular com trabeculados ósseos imaturos, delimitados por osteoblastos proeminentes em forma de halo.

O tratamento cirúrgico proporciona alívio rápido dos sintomas, tem uma elevada taxa de sucesso (88 a 100\%), poucas recidivas, permite o exame histológico e envolve a remoção cirúrgica de todo o nicho. Diversas técnicas foram desenvolvidas, dentre elas a ressecção em bloco do osteoma osteoide e do osso adjacente e a curetagem do nicho com remoção de parte do osso reativo circundante; entretanto, a principal dificuldade consiste na localização exata do nicho. ${ }^{7-9}$ A ressecção cirúrgica em bloco guiada por gramaprobe propõe fim para este contratempo, reduzindo o tamanho da incisão, aumentando a probabilidade de remover todo o nicho e possibilidade de confirmação diagnóstica por anatomopatológico. ${ }^{6}$

O método de ressecção guiada por gamaprobe é realizada em dois tempos; o primeiro tempo praticado no centro de medicina nuclear, onde o paciente é submetido à cintilografia óssea com tecnécio com posterior demarcação da topografia acometida baseado nas imagens obtidas. A segunda etapa acontece no centro cirúrgico, sendo realizada varredura com auxílio de sonda de raios gama. ${ }^{10}$

Entretanto, atualmente, a técnica de ablação por radiofrequência é considerada o procedimento de destruição percutânea do nicho mais aceita, principalmente por possuir significativas vantagens em relação às demais, demonstrando ser uma técnica segura e eficaz, adequada à maioria dos pacientes, possível de efetuar na maioria dos centros e de rápida execução. ${ }^{11,12}$

O presente trabalho tem por objetivo descrever a técnica de ressecção do tumor ósseo guiado por gamaprobe e avaliar a melhora do quadro álgico no pós-operatório.

\section{Materiais e Métodos}

Estudo prospectivo realizado com 14 pacientes diagnosticados com osteoma osteóide submetidos a ressecção guiada por gamaprobe em um hospital credenciado entre maio de 2010 e maio de 2017 mediante análise de prontuários, escala analógica de dor pré- e pós-operatória e grau de satisfação do paciente. Dos pacientes analisados, 11 (78,47\%) eram do sexo masculino e $3(21,43 \%)$ do sexo feminino, com idade variando de 3 a 41 anos (-Tabela 1).

Devido ao caráter do estudo, foi utilizado um Termo de Consentimento Livre e Esclarecido e uma ficha de dados com aspectos relevantes como data da cirurgia, topografia, escala analógica de dor pré- e pós-operatória e técnica utilizada. 

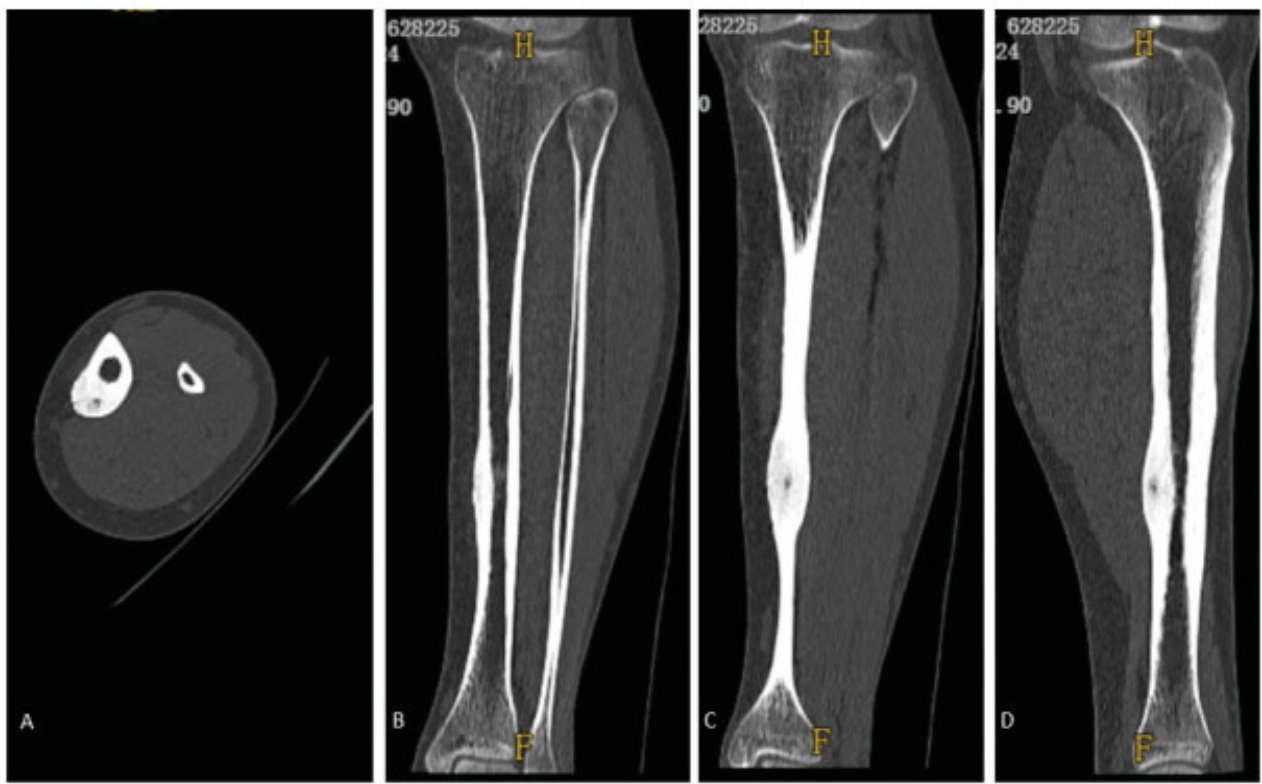

Fig. 2 Cortes tomograficos da perna, $A=$ corte axial, $B=$ corte coronal, $C=$ corte coronal em um plano mais posterior, $\mathrm{D}=$ corte sagital, demonstrando espessamento da cortical lateral da tíbia, com presença de região com menor densidade na região justa-cortical posterolateral, compatível com o nicho.

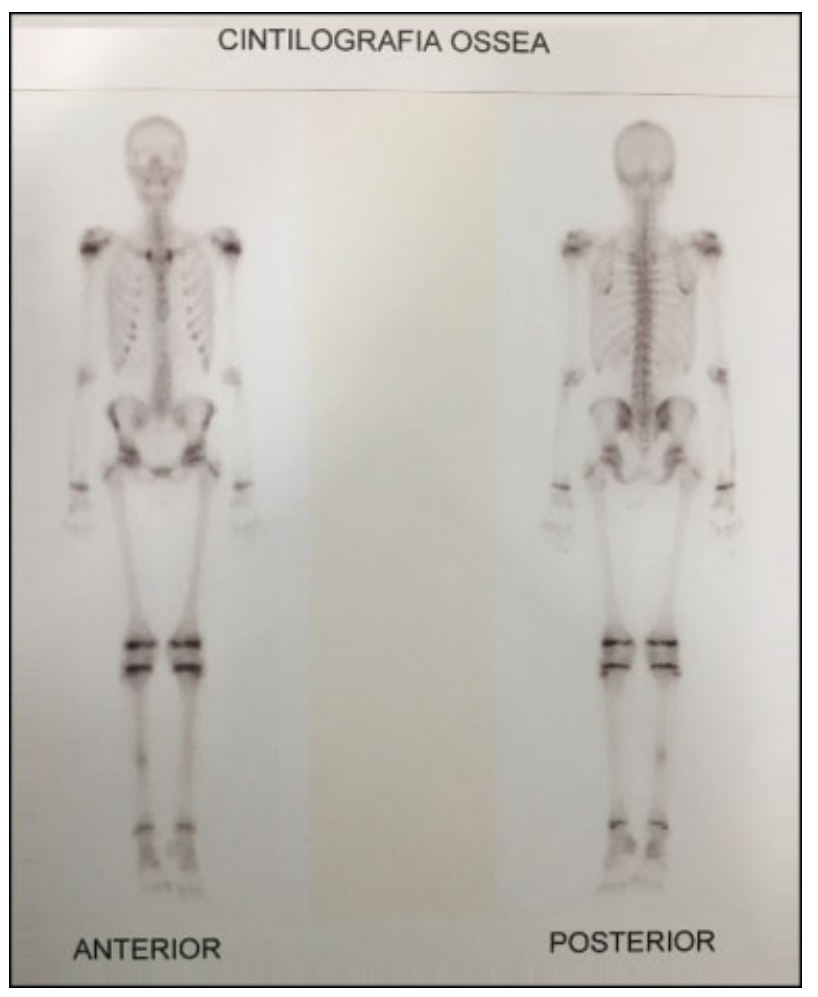

Fig. 3 Imagem de cintilografia de fluxo com tecnécio 99, com aumento de captação na região diafisária da tíbia direita.

Tabela 1 Distribuição dos pacientes conforme gênero e suas respectivas porcentagens

\begin{tabular}{|l|l|l|}
\hline Gênero & Masculino & Feminino \\
\hline Total $(\boldsymbol{n}=\mathbf{1 4})$ & $n=11$ & $n=3$ \\
\hline Porcentagem $(\%)$ & $78,47 \%$ & $21,43 \%$ \\
\hline
\end{tabular}

Foram excluídos pacientes que foram submetidos a tratamento com outra técnica cirúrgica.

\section{Descrição da Técnica}

Os pacientes do presente estudo foram submetidos à cirurgia sob anestesia geral ou bloqueio regional, no centro cirúrgico.

No dia da cirurgia, os pacientes foram submetidos à primeira etapa no centro de medicina nuclear, onde foi realizada a cintilografia óssea com fluxo de tecnécio 99 com injeção de $1110 \mathrm{MBq}$ de MDP- ${ }^{99 \mathrm{~m}} \mathrm{Tc}$ para os adultos; nas crianças, a dose foi corrigida para idade e peso. São obtidas imagens $\sim 2$ horas após a injeção do radioisótopo. Ainda com auxílio da cintilografia de fluxo com tecnécio 99 (-Figura 4), são demarcados, na pele, os locais de maior captação pela sonda de raios gama - gamaprobe ( Figuras 5A e 5B - para auxiliar na incisão no centro cirúrgico. Após realizada a demarcação, o paciente é encaminhado para o centro cirúrgico para realização da segunda etapa, onde são anestesiados com técnica pertinente para cada caso e para realizar a ressecção. A incisão é baseada nos exames préoperatórios e nas marcações que foram possibilitadas pela leitura da radiação emitida pelo nicho. Após realizado acesso cirúrgico e localizado o suposto local da lesão, a sonda é posicionada a formar um ângulo de $90^{\circ} \mathrm{com}$ a superfície óssea, a fim de identificar o nicho, ponto com maior emissão de radiação. Ao identificar o ponto de maior captação, realiza-se a ressecção em bloco.

Após coleta do material que é enviado para análise anatomopatológica, realiza-se ampliação e curetagem de cerca de 1 a $2 \mathrm{~mm}$ da cavidade, seguido de limpeza com soro fisiológico a 0,9\% (-Figura 5). Utiliza-se novamente o gamaprobe na área ressecada confirmando decréscimo da captação. A seguir, sutura dos tecidos por planos. A enxertia óssea é realizada conforme necessidade do sítio cirúrgico. 


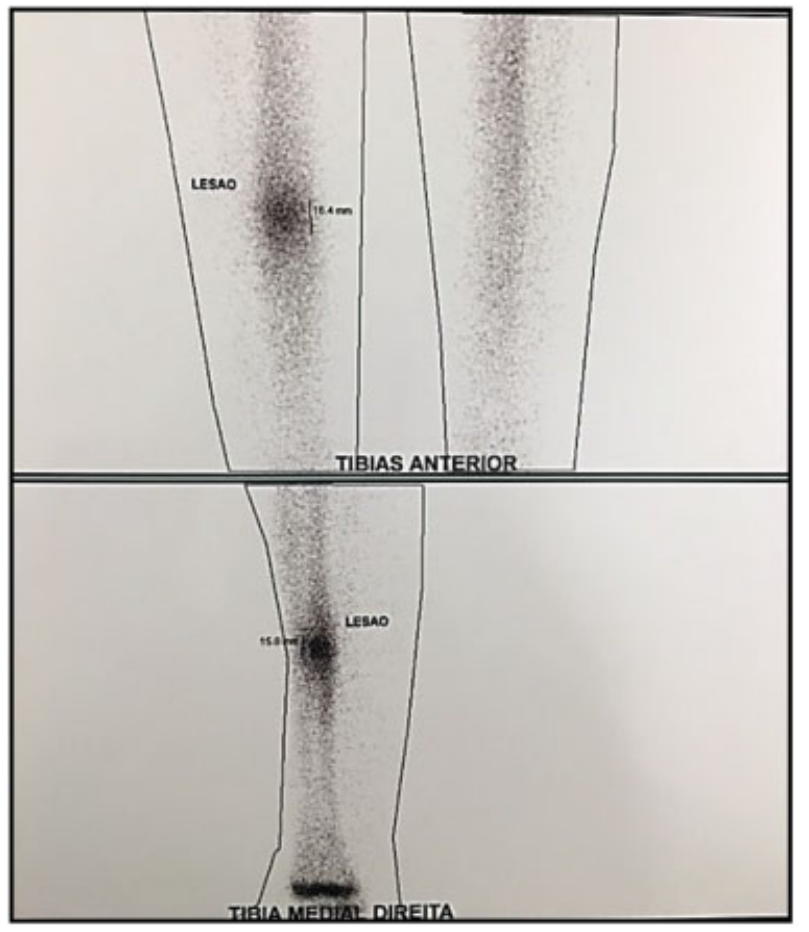

Fig. 4 Imagem focalizada na região da tíbia na posição anterior e de medial para lateral, onde observa-se aumento da captação na região posterolateral - local da demarcação da pele.
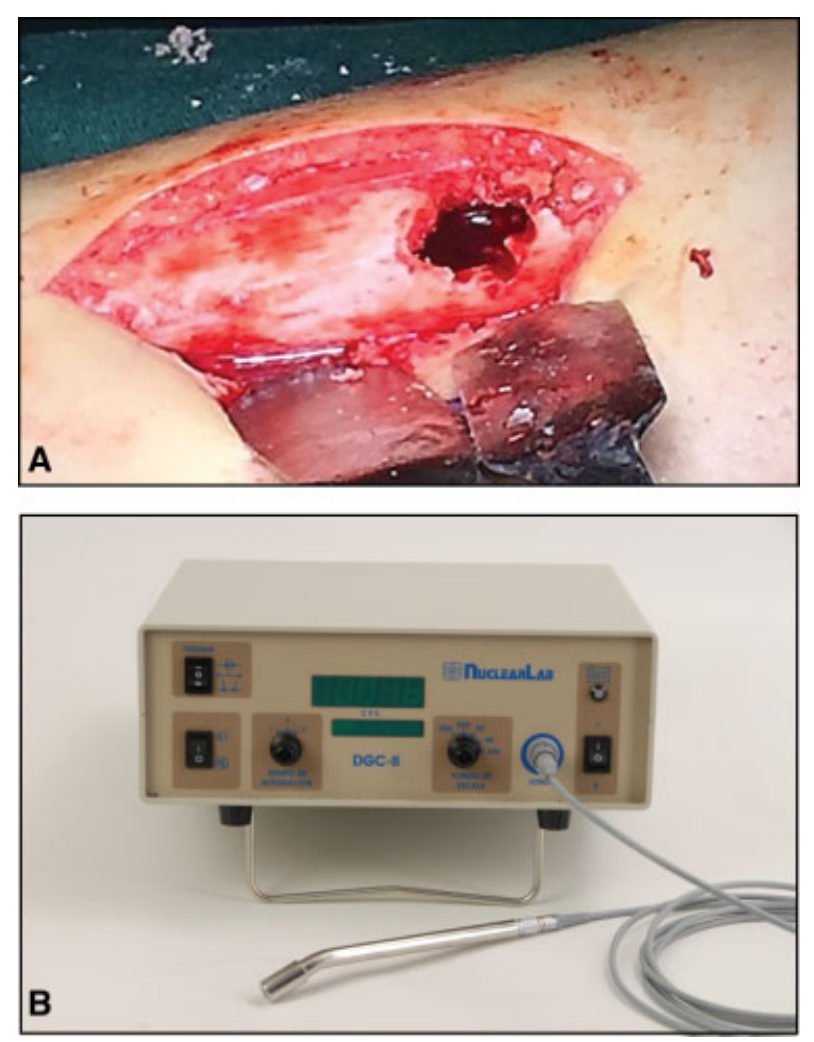

Fig. 5 (A) Incisão no local da marcação da pele com ressecção em bloco guiada pelas imagens prévias e pela captação do gamaprobe. Após ressecção, foi realizado ampliação da lesão com curetagem. (B) Equipamento do gramaprobe utilizado no intraoperatório para identificar as lesões previamente demarcadas pela medicina nuclear.
Tabela 2 Avaliação do quadro álgico nos pacientes avaliados e respectivas porcentagens

\begin{tabular}{|l|l|l|l|}
\hline & Alívio Total & $\begin{array}{l}\text { Alívio } \\
\text { Parcial }\end{array}$ & $\begin{array}{l}\text { Sem } \\
\text { mudança }\end{array}$ \\
\hline Total $(n=14)$ & $n=14$ & 0 & 0 \\
\hline Porcentagem $(\%)$ & $100 \%$ & $0 \%$ & $0 \%$ \\
\hline
\end{tabular}

Tabela 3 Distribuição segmentar do acometimento dos pacientes e respectivas porcentagens

\begin{tabular}{|l|l|l|}
\hline & Membro Inferior & Membro Superior \\
\hline Total $(n=14)$ & $n=11$ & $n=3$ \\
\hline Porcentagem (\%) & $78,47 \%$ & $21,43 \%$ \\
\hline
\end{tabular}

Tabela 4 Distribuição dos ossos acometidos e respectivas porcentagens

\begin{tabular}{|l|l|l|}
\hline Segmento acometido & Total $(\mathbf{n}=\mathbf{1 4})$ & Porcentagem (\%) \\
\hline Úmero & $n=1$ & $7,14 \%$ \\
\hline Tíbia & $n=6$ & $42,85 \%$ \\
\hline Fíbula & $n=1$ & $7,14 \%$ \\
\hline Fêmur & $n=4$ & $28,57 \%$ \\
\hline Escápula & $n=2$ & $14,3 \%$ \\
\hline
\end{tabular}

No pós-operatório, após recuperação anestésica, é possível avaliar o sucesso do procedimento, correlacionando o mesmo com alívio da dor. Se houver alívio total da dor referida no período antecedente à cirurgia, infere-se que foi realizada ressecção total do nicho.

\section{Resultados}

Dos 14 pacientes 11 (78,47\%) eram do sexo masculino e 3 (21,43\%) do sexo feminino, Todos os $14(100 \%)$ foram submetidos à técnica de ressecção guiada por gamaprobe. Destes, todos apresentaram alívio completo dos sintomas (100\%) (-Tabela 2). Onze (78,47\%) estavam localizados nos membros inferiores, e $3(21,43 \%)$ nos membros superiores ( - Tabela 3). 0 osso mais acometido foi a tíbia ( $n=6 ; 42,85 \%$ ), seguido do fêmur $(n=4 ; 28,57 \%)$ e demais ossos em menor frequência (-Tabela 4).

A faixa etária mais acometida foi na $3^{a}$ década de vida $(n=5 ; 35,7 \%)$, com a mesma periodicidade na $2^{2}$ e 4 a décadas $(n=3 ; 21,4 \%)$, e menor frequência na $1^{\text {a }}$ década $(n=2 ; 14,3 \%)$

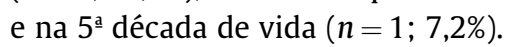

A prevalência do osteoma osteóide apresentou um pico na $2^{2}$ década de vida, compreendendo a faixa etária dos 21 aos 30 anos. Os casos da patologia ente 0 e 10 anos referem-se a $\sim$ $15 \%$ da nossa causuística, bem como a faixa etária dos 11 aos 20 anos contemplam 20\%; dos 31 aos 40 anos, o número de casos atinge igualmente $20 \%$, e a última faixa etária dos pacientes selecionados, 41 a 50 anos, engloba $\sim 5 \%$ da amostra. (-Figura 6) 


\section{Relação Idade x Número de Casos}

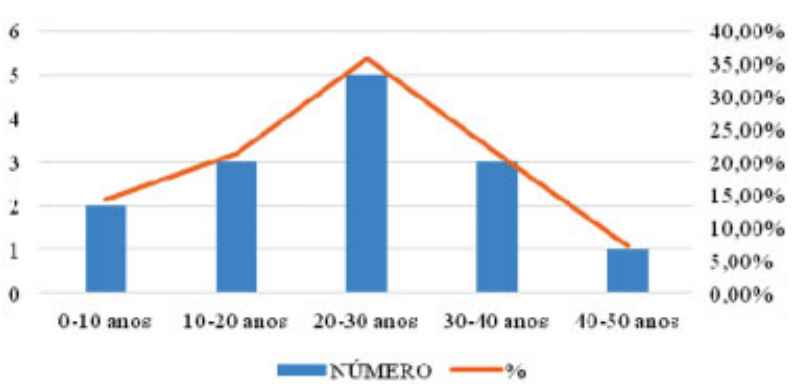

Fig. 6 Relação entre idade e número de casos dos pacientes analisados e a respectiva porcentagem.

\section{Discussão}

O osteoma osteóide é descrito na literatura como uma lesão tumoral benigna frequente, que acomete principalmente homens jovens, sendo mais comum durante a $2^{\underline{a}}$ e $3^{\underline{a}}$ décadas de vida, dados estes confirmados no presente estudo. ${ }^{6,13} \mathrm{O}$ diagnóstico da lesão é clínico, corroborado por radiografia comum e TC. A confirmação do diagnóstico é dada pelo exame anatomopatológico, porém ele não se faz necessário para diagnóstico, visto que, devido ao pequeno tamanho do nicho, a biópsia excisional já seria curativa.

A técnica cirúrgica de excelência não é consenso na literatura, devido à sua pequena casuística e à limitação dos estudos de descrição. Ehara et al $^{14}$ e Campanacci et al $^{12}$ comparam duas técnicas cirúrgicas para resseção do osteoma osteóide, porém não comparam técnicas para localização do nicho sendo este o impasse da abordagem cirúrgica. Este fato levou a comunidade científica a procurar novas técnicas para identificar o nicho e, assim, diminuir a morbidade e obter resultados mais consistentes e satisfatórios.

A técnica de localização com auxílio da tomografia computadorizada foi desenvolvida com este intuito, porém ainda apresentava alguns impasses. $O$ fato de o paciente ser submetido a dois procedimentos invasivos, a marcação durante tomografia com agulha e procedimento cirúrgico definitivo seriam pontos negativos da mesma, o que não ocorre na marcação com cintilografia com fluxo com tecnécio 99. ${ }^{6,14-16}$

Nos últimos anos, técnicas percutâneas tornaram-se o tratamento de escolha do osteoma osteóide, com taxas de sucesso de entre 80 e $100 \%{ }^{9}$ incluindo a excisão percutânea do nicho e a destruição do mesmo por injeção de etanol, crioablação, ablação a laser ou radiofrequência. ${ }^{11,12,17}$ Por serem minimamente invasivas, são seguras, realizadas com anestesia local, regional ou sedação, permitem a destruição do nicho sem excisão significativa do osso adjacente, e podem ser realizadas em ambulatório ou em internamentos de curta duração. A recuperação é rápida, e tanto as complicações quanto as recidivas são raras, constituindo assim uma alternativa às técnicas convencionais. ${ }^{11,18,19}$

As técnicas percutâneas têm aplicação limitada nos osteomas osteóides recorrentes, principalmente os de localização na coluna, próximos a estruturas nervosas ou em ossos muito pequenos. ${ }^{5,17}$ Nesses casos, as técnicas de ressecção aberta são a melhor escolha. Além disso, o uso de trefinas, apesar de percutâneas, são procedimentos mais agressivos e relacionados a fraturas devido ao fato de aumentar a fragilidade cortical na diáfise dos ossos longos. ${ }^{20,21}$

A técnica de ressecção guiada por radioisótopo é uma técnica segura, pode ser realizada em qualquer localidade que disponha de um centro de medicina nuclear, diminui o número de procedimentos para o paciente, o tamanho da incisão (não avaliado no presente trabalho, mas possível para uma próxima análise), guia mais fidedignamente o local da incisão e localiza com maior precisão o nicho tumoral no intraoperatório. A confirmação diagnóstica pode ser conferida pela avaliação histológica através de exame anatomopatológico; porém, conforme Sim et $\mathrm{al}^{22}$ um resultado negativo não exclui o diagnóstico correto, haja visto que o material do nicho é friável e pode ser muitas vezes, devido seu tamanho pequeno, mal selecionado na hora da coleta.

Visto que a causa da dor intensa é a presença do nicho, leva-se em consideração o sucesso da técnica devido à taxa de $100 \%$ de alívio da dor no pós-operatório. Outros parâmetros da literatura que se confirmaram foram a prevalência de acometimento em ossos longos e do membro inferior. ${ }^{4}$

\section{Conclusão}

A técnica de ressecção guiada por radioisótopo configura uma técnica segura e resolutiva, ainda que não seja o padrão ouro utilizado na atualidade, sendo considerada uma opção adequada para o tratamento do osteoma osteóide com 100\% de alívio da dor no pós-operatório imediato.

Embora existam desvantagens da ressecção cirúrgica em relação às técnicas minimamente invasivas, como uma maior incisão cirúrgica e necessidade de uma íntima relação com medicina nuclear, sobretudo no que tange ao uso da cintilografia, é imprescindível ressaltar que as vantagens apresentadas como diminuição de custo, precisão do uso da cintilografia e obtenção da peça para análise anatomopatológica, fazem desta técnica uma excelente escolha. Além das características acima citadas, acredita-se que pela precisão da técnica, obtenha-se o procedimento cirúrgico com menor tempo cirúrgico, o que poderá ser avaliado em um possível seguimento do trabalho.

\section{Conflito de Interesses}

Os autores declaram não haver conflito de interesses.

\section{Referências}

1 Canale ST, Beaty JH. Campbell cirurgia ortopédica. 12ed. Rio de Janeiro: Elsevier; 2017

2 Jesus-Garcia R. Diagnóstico e tratamento de tumores ósseos. Rio de Janeiro: Elsevier; 2013

3 Jaffe HL. Osteoid osteoma. A benign osteoblastic tumor composed of osteoid and atypical bone. Arch Surg 1935;31(05):709-728

4 Sousa M, Freitas D, Cardoso P. Osteoma Osteóide? Rev Port Ortop Traumatol 2014;22(01):135-141

5 Lee EH, Shafi M, Hui JH. Osteoid osteoma: a current review. J Pediatr Orthop 2006;26(05):695-700 
6 Endo RR, Gama NF, Nakagawa SA, Tyng CJ, Chung WT, Pinto FFE. Osteoid osteoma - radiofrequency ablation treatment guided by computed tomography: a case series. Rev Bras Ortop 2017;52(03): 337-343

7 Rankine JJ. The diagnosis and percutaneous treatment of osteoid osteomas. Curr Orthop 2007;21(06):464-470

8 Papathanassiou ZG, Megas P, Petsas T, Papachristou DJ, Nilas J, Siablis D. Osteoid osteoma: diagnosis and treatment. Orthopedics 2008;31(11):1118

9 Nielsen GP, Rosenberg AE. Bone-forming tumors. In: Folpe AL, Inwards CY. Bone and Soft Tissue Pathology: a volume in the series foundations in diagnostic pathology. Philadelphia: Churchill Livingstone; 2010:309-328

10 Etchebehere M, Etchebehere EC, Reganin LA, Amstalden EM, Cliquet A Jr, Camargo EE. Intraoperative localization of an osteoid-osteoma using a gamma probe. Int Orthop 2004;28(06):379-383

11 Vigorita VJ. Orthopaedic pathology. 2nd ed. Philadelphia: Lippincott Williams \& Wilkins; 2008:339-348

12 Campanacci M, Ruggieri P, Gasbarrini A, Ferraro A, Campanacci L. Osteoid osteoma. Direct visual identification and intralesional excision of the nidus with minimal removal of bone. J Bone Joint Surg Br 1999;81(05):814-820

13 David A, Rios AR, Tarragô RP, Oliveira GK, Garzela MM, Oliveira RK. Excisão de osteoma osteóide por trefina orientada pela tomografia computadorizada. Rev Bras Ortop 1997;32(05): $396-400$
14 Ehara S, Rosenthal DI, Aoki J, Fukuda K, Sugimoto H, Mizutani H, et al. Peritumoral edema in osteoid osteoma on magnetic resonance imaging. Skeletal Radiol 1999;28(05):265-270

15 Gangi A, Dietemann JL, Gasser B, Mortazavi R, Brunner P, Mourou $\mathrm{MY}$, et al. Interstitial laser photocoagulation of osteoid osteomas with use of CT guidance. Radiology 1997;203(03):843-848

16 Lindner NJ, Ozaki T, Roedl R, Gosheger G, Winkelmann W, Wörtler K. Percutaneous radiofrequency ablation in osteoid osteoma. J Bone Joint Surg Br 2001;83(03):391-396

17 Sluga M, Windhager R, Pfeiffer M, Dominkus M, Kotz R. Peripheral osteoid osteoma. Is there still a place for traditional surgery? J Bone Joint Surg Br 2002;84(02):249-251

18 Yang WT, Chen WM, Wang NH, Chen TH. Surgical treatment for osteoid osteoma - experience in both conventional open excision and CT-guided mini-incision surgery. J Chin Med Assoc 2007;70 (12):545-550

19 Moser T, Buy X, Goyault G, Tok C, Irani F, Gangi A. [Image-guided ablation of bone tumors: review of current techniques]. J Radiol 2008;89(04):461-471

20 Yildiz Y, Bayrakci K, Altay M, Saglik Y. Osteoid osteoma: the results of surgical treatment. Int Orthop 2001;25(02):119-122

21 Parlier-Cuau C, Champsaur P, Nizard R, Hamze B, Laredo JD. Percutaneous removal of osteoid osteoma. Radiol Clin North Am 1998;36(03):559-566

22 Sim FH, Dahlin CD, Beabout JW. Osteoid-osteoma: diagnostic problems. J Bone Joint Surg Am 1975;57(02):154-159 